\title{
Cancer therapy leading to state of cancer metabolism depression for efficient operation of small dosage cytotoxic drugs
}

\author{
Ponizovskiy $\mathrm{MR}^{1, *}$ \\ ${ }^{1}$ Head of Laboratory Biochemistry and Toxicology, Kiev Regional p/n Hospital, Kiev, Ukraine
}

\begin{abstract}
"Prolonged medical starvation" as the method of cancer therapy was borrowed from folk healers Omelchenko A and Breuss R. Author was convinced in efficiency of this method of cancer treatment via examination of cured patients and on own experience. The mechanism of this method of cancer therapy operates via Warburg effect targeting that promotes efficient cancer treatment with small cytotoxic drugs. Just it was described the mechanism of Warburg effect as well as mechanism transmutation of mitochondrial function in cancer metabolism which are exhibited in connection with operation of described method cancer therapy. There were described the biochemical and biophysical mechanisms of formations resistance to some cytotoxic drugs and recurrence cancer disease after disease remission which occur sometimes as result of treatment with great dosage of cytotoxic drugs. Also it was described the benefits of use the method "Prolonged medical starvation" with decreased dosage of cytotoxic drugs for cancer treatment. The significance of this work that it was substantiated the mechanism operation of combination "Prolonged medical starvation" with small dosages cytotoxic drugs of cancer treatment, which mechanism leads to prevention recurrence cancer disease and resistance to anticancer drugs in comparison with intensive anticancer chemotherapy with great dosages of cytotoxic drugs in cancer therapy. Also the offered concepts of cancer therapy mechanism gave possibility to explain mechanisms of some results of experiments eliminating the doubts of the authors these experiments.
\end{abstract}

Keywords: cancer therapy; cytostatic effect; tyrosine kinase enzymes; glycolysis inhibition; nuclear DNA; ROS; mtDNA

\section{Introduction}

Austrian folk healer Rudolf Breuss 1992 described the positive results of oncologic diseases treatment using "Long Starvation during 42 days" [1, 2]. However the mechanisms of operating of this method treatment could not be explained by the folk healer. Having borrowed from the folk healers Omelchenko A and Breuss R. the method treatment of the oncologic patients using "Prolonged medical starvation 42-45 days", having tested the positive results of this method of treatment, it was explained the mechanism of this method treatmentand wassubstantiated this method treatment, using the offered concept of Warburg effect mechanism [3-5]. Author was convinced of efficiency of this method treatment by the meetings with cured patients, who obtained the folk healer Omelchenko treatment, and determined on own experience efficiency of treatment the ill man with the incurable cancer stage as well as testing the factors of this method treatment as the experiments on itself. The changes, occurring in an organism, have been analysed by the author from the point of view of biochemistry and biophysics. So taking into account that an organism maintain stability Internal Energy (IE) (temperature $36.6^{\circ} \mathrm{C}$ etc.) and Internal Medium (IM) (concentration substances in blood and neurolymph) as in norm as well as in pathology, there were maintained stability also blood sugar stuff during this method treatment. The scientific explanation of the mechanism action of this method treatment was given using the concept of Warburg effect mechanism [3-5] (Figure 1). Also both the concept of Warburg effect mechanism and the concept of this method treatment mechanism were arisen by studies and elucidations of the mechanisms maintenance stability IE and IM in an organism and in cells of an organism as in normal stationary state as well as in quasi-stationary pathologic state of an organism [6-8]. According to first law of thermodynamics, stationary state of open nonequilibrium non-linear thermodynamic system of an able-bodied organism and also quasi-stationary state of open non-equilibrium non-linear thermodynamic system of a sick organism are characterized by stability of IE $(\Delta U)$ (stable temperature $36.0^{\circ} \mathrm{C}-36.9^{\circ} \mathrm{C}$ by which all enzymes operate etc.) $[6,7]$. Stability of IE determines stability of

\footnotetext{
*Corresponding author: M. R. Ponizovskiy, Head of Laboratory Biochemistry and Toxicology, Kiev Regional p/n Hospital, Kiev, Ukraine. Tel.: (49911)-653-78-11; Email: ponis@online.de

Received 15 January 2015 Revised 14 March 2015 Accepted 22 March 2015 Published 29 March 2015

Citation: Ponizovskiy MR. Cancer therapy leading to state of cancer metabolism depression for efficient operation of small dosage cytotoxic drugs. J Cancer Res Ther. 2015; 3(3):26-43. DOI:10.14312/2052-4994.2015-5

Copyright: () 2015 Ponizovskiy MR. Published by NobleResearch Publishers. This is an open-access article distributed under the terms of the Creative Commons Attribution License, which permits unrestricted use, distribution and reproduction in any medium, provided the original author and source are credited.
} 
IM (constant concentration substances in blood and in neurolymph). It was considered the mechanism of remote cellular reactions due to cellular capacitors operations, which transit into contact biochemical reaction in immune defensive mechanism of an organism. Unlike able-bodied cells, normal cells don't react via resonance waves to cancer cells because chemical potentials of cancer cells are unrelated to chemical potentials of normal cells (Figures $2,3)$. Just the new method of cancer therapy via targeting of Warburg effect leads to cancer depression that gives opportunity to use considerably decreased dosage of cytotoxic drugs for efficient cancer treatment $[9,10]$. Such approach to cancer therapy does not damage immune and hormonal systems versus official up-to-date methods of chemotherapy. Thus the explanations mechanisms stability of cellular internal energy, as in normal stationary state of able-bodied cells and as well as in pathologic quasistationary states of cancer cells, gave possibility to explain mechanism operation "Prolonged medical starvation" with considerably decreased of cytotoxic drugs' dosage [10]. All of it gave possibility to substantiate the advantage this method of cancer therapy, which is based on Warburg effect targeting, over some up-to-date methods of cancer therapy $[4,5,10]$. Author gave explanation both the mechanism decrease of medical drugs efficiency and mechanism palindromia of cancer recurrence after some medical remissions. Also the author noted that the offered method cancer therapy should be put into practice after detail clinic trials. Besides, there were explained mechanisms of some experiments, eliminating doubts which were expressed by the authors of these experiments.

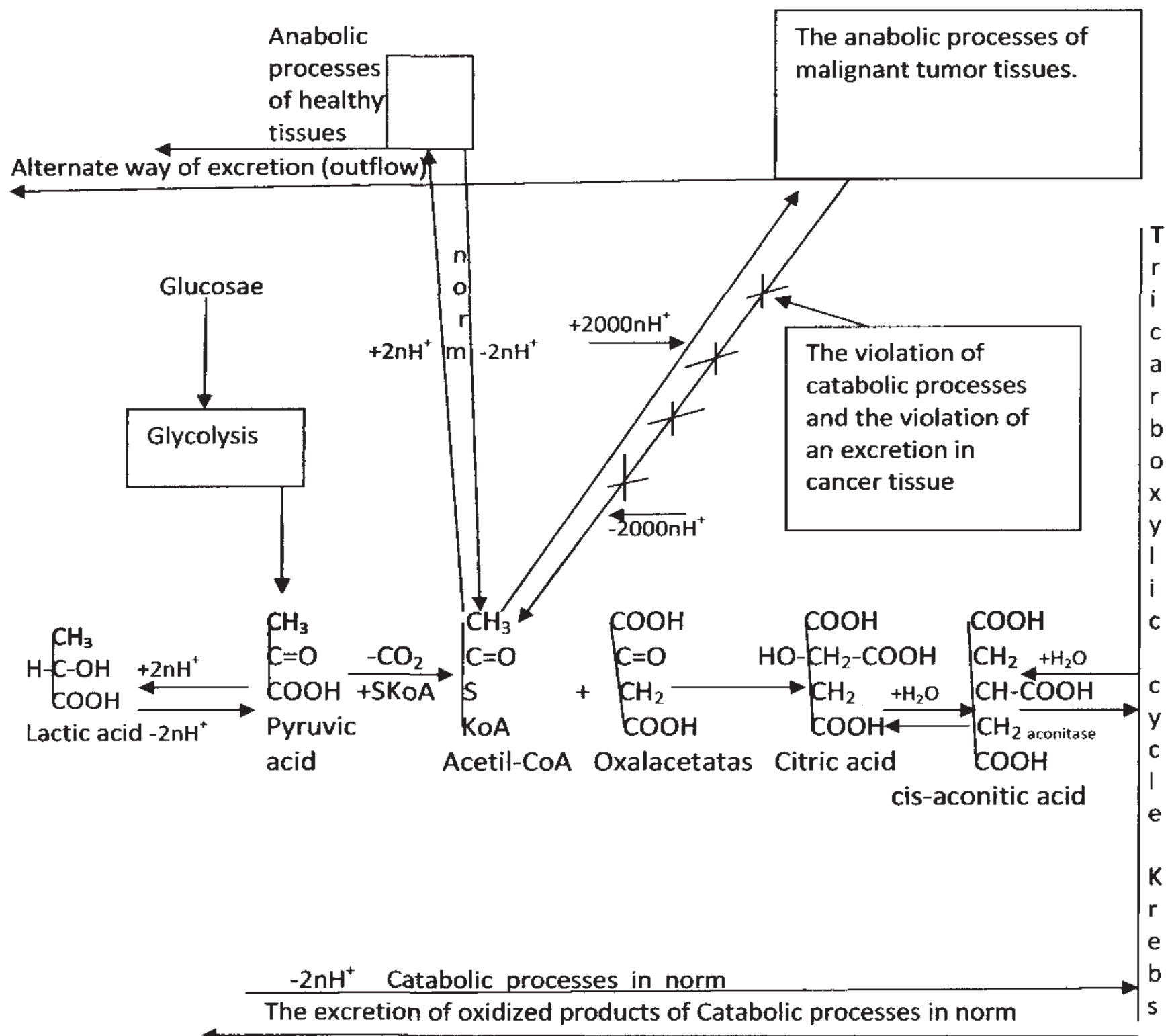

Figure 1 The metabolism of a malignant tumor tissue and of a normal tissue.

Note: a) Nodal point of bifurcation anabolic and catabolic processes, b) Huge anabolic processes with huge consumption of energy and Acetyl-CoA for anabolic processes leading to overloading "Nodal point of bifurcation anabolic and catabolic processes" [NPBac] in cancer tissue, c) Moderate metabolic processes displaying balance anabolic and catabolic processes in able-bodied tissue, d) Alternative excretion of high-molecular substances within the structure rejected cells and the violation of excretion substances via oxidative processes due to suppression of catabolic oxidative processes in cancer tissue, e) Accumulation of energy into lactic acid for anabolic processes, f) Normal excretion substances via catabolic oxidative processes in able-bodied tissue. 


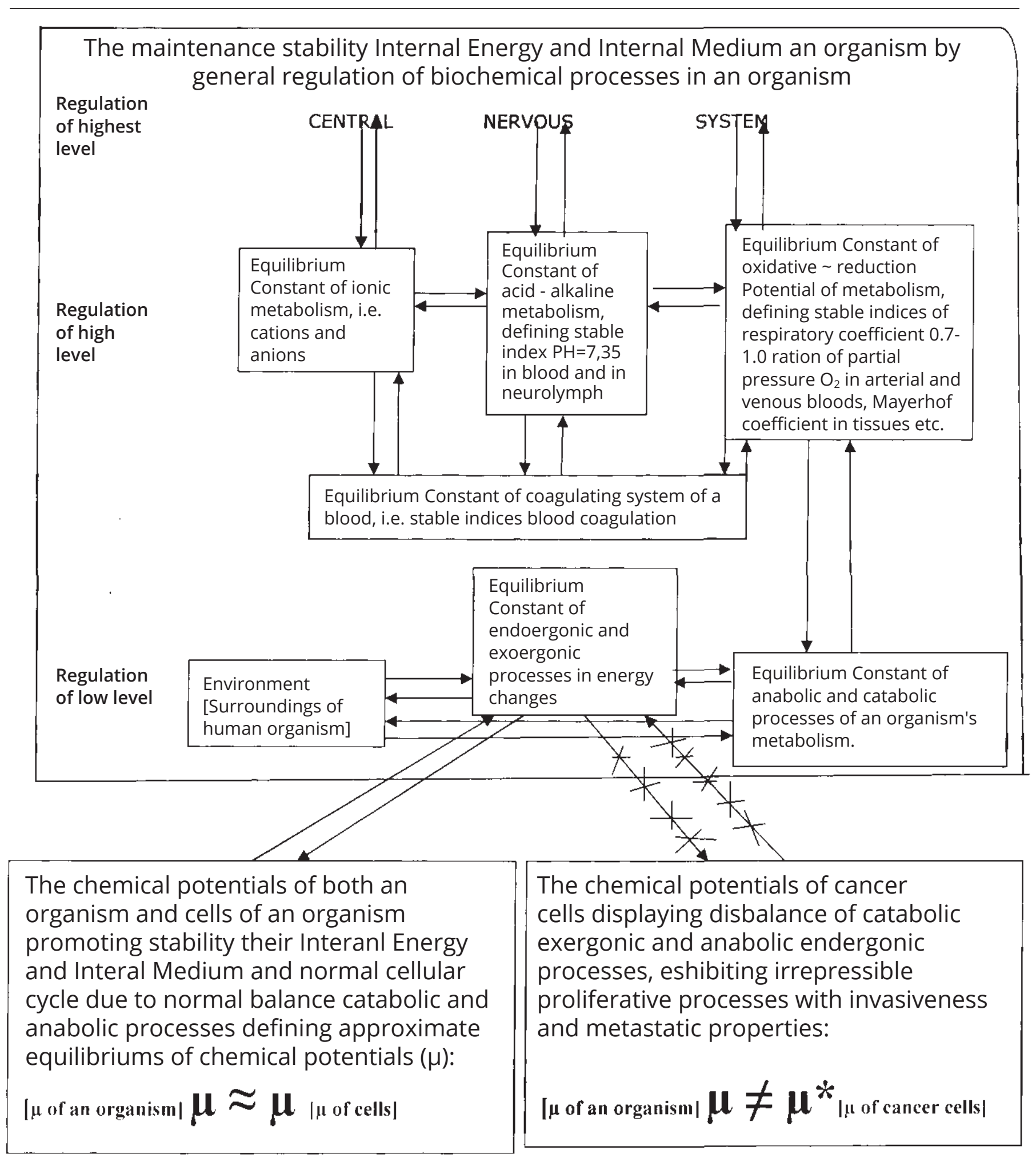

Figure 2 The influences general regulation biochemical processes on Internal Energy determining stability internal chemical potentials of an organism ( $\mu$ ), normal cells $(\mu)$ and cancer cells $\left(\mu^{*}\right)$.

Note: a) General regulation biochemical processes exhibits mutual influences between Low level Regulation, High level Regulation and Highest level Regulation, b) Low level Regulation consists of "Equilibrium Constants of balance endoergonic and exoergonic processes of energy exchange" and "Equilibrium Constants of balance anabolic and catabolic processes of metabolism" which cause mutual influences one another, c) Low level Regulation is subjected to Environment influences and effects against Environment influences for maintenance stability Internal Energy and Internal Medium as an organism as well as cells of an organism, d) High level Regulation consists of mutual interacted "Equilibrium Constants of ionic metabolism", "Equilibrium Constants of acid - alkaline metabolism", "Equilibrium Constants of oxidative - reductive Potentials of metabolism" and "Equilibrium system of coagulating system", which cause mutual influences with "Equilibrium Constants of coagulating system of a blood", e) The Regulation both Low level Regulation and High level Regulation is occurred via mutual influences between "Equilibrium Constants of oxidative - reductive Potentials of metabolism" of High level Regulation and "Equilibrium Constants of anabolic and catabolic processes of metabolism" of Low level Regulation, f) Highest level Regulation is presented by CENTRAL NERVOUS SYSTEM, g) General regulation biochemical processes creates chemical potential an organism $(\mu)$ which mutual influences on one another with related chemical potentials of cells of an organism $(\mu), \mathrm{h})$ Chemical potentials of cancer cells $\left(\mu^{*}\right)$ were created by penetrating oncogens that destroys interactions between chemical potentials of an organism $(\mu)$ and chemical potentials of cancer cells $\left(\mu^{\star}\right)$. 


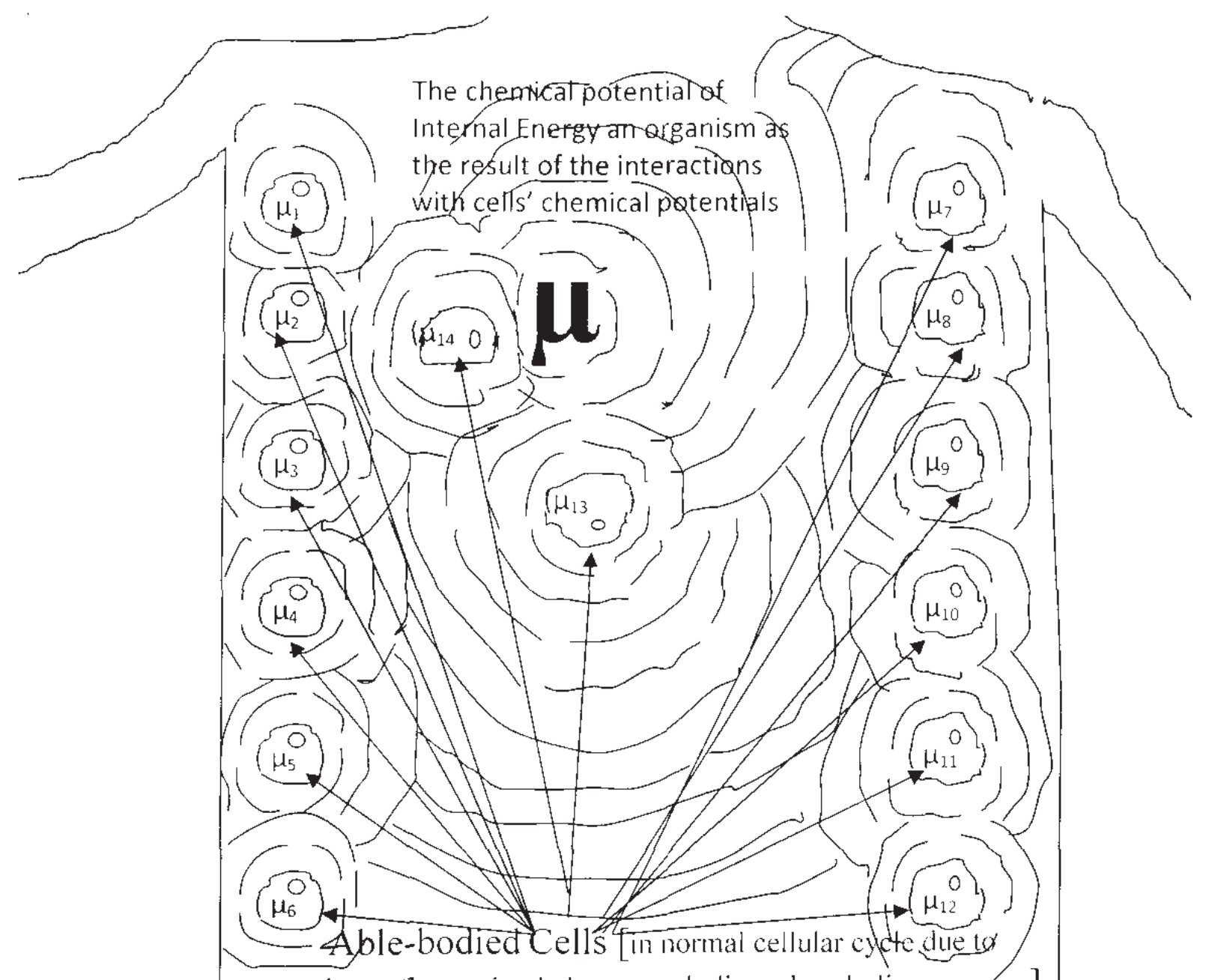

moderate fluctuating balance catabolic and anabolic processes]

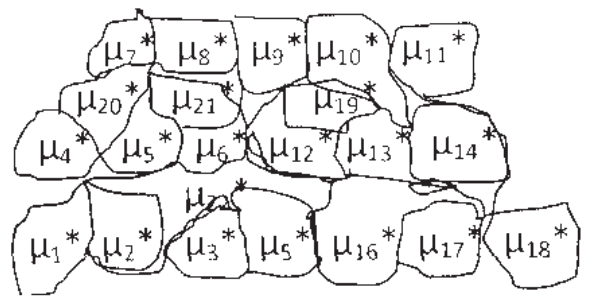

Cancer cells [exessive shift catabolic and anabolic processes into anabolic processes, exhibiting irrepressible proliferative processes with invasiveness and metastatic properties]

Figure 3 Balance Internal Energy both cells and an organism due to their chemical potentials $(\mu)$ promoting operation resonance waves of cellular capacitors and disbalance of chemical potentials $\left(\mu^{\star}\right)$ cancer cells.

Note: a) Chemical potential of an organism $(\mu)$ is the indicator of stability Internal Energy an organism, b) Chemical potential of an organism ( $\mu$ ) defines related chemical potentials of cells an organism $(\mu)$ as the indicators of stability Internal Energy of cells an organism, c) Resonance waves between an organism and cells of an organism are produced by cellular capacitors which reflect interactions between cells of an organism and between an organism and cells of an organism due to related chemical potentials of cells an organism $(\mu)$, d) Chemical potentials of cancer cells ( $\left.\mu^{*}\right)$ are the unrelated potentials to chemical potentials of an organism $(\mu)$ and chemical potentials of an organism's cells $(\mu)$ that results in absent cohesive resonance waves joining them and absent regulating influences of an organism showing autonomous development of cancer cells via excessive expression of proliferative processes. 


\section{Concept of Warburg effect mechanism for substantiation cancer therapy via Warburg effect targeting (Figure 1)}

Highlight of Warburg effect concept [3] (Figure 1): As the result of oncogenes operation causing enormous anabolic processes in cancer tissue and the enormous consumption of energy and Acetyl-CoA for anabolic (biosynthetic) processes, it takes place the overload of "nodal point of bifurcation anabolic and catabolic processes" (NPBac) because of the remained lack of Acetyl-CoA for catabolic oxidative processes. Such shift into anabolic processes and lack Acetyl-CoA causes partial suppression of catabolic processes in cancer tissue, some of which remain for cancer cells survival. The increase of lactic acids production is the necessary endergonic mechanism accumulation of energy for huge anabolic processes in condition glycolysis metabolism and enormous consumption of energy for anabolic processes in cancer tissue [3]. Just unlike Pasteur effect [Incompatibility glycolysis with aerobic oxidation] in normal tissue, Warburg effect exhibits aerobic glycolysis [combination aerobic oxidation with glycolysis (increase lactic acids-crucial marker glycolysis)] in cancer tissue metabolism that leads to apoptosis resistance of cancer cells. Therefore there were hot busted mitochondria [the main center of aerobic oxidation] in cancer cells or were restored aerobic function due to acquisition mitochondria in host of cancer cells in event of loss mitochondria according to new research [11]. This concept gives possibility to explain as Warburg effect mechanism, elucidating distinction between mechanisms of Pasteur effect and of Warburg effect, as well as mechanisms of "Contact inhibition of propagating cells in norm" and of "Absence of contact inhibition of propagating cells in malignant tumor" using Theorell formula [3]. Besides, the offered concept gives possibility to explain the mechanisms of irrepressible tumor growth, non-healed cancer ulcer and mechanism of metastases formation [3] (Figure 1). The offered concept of Warburg effect mechanism gives possibility to explain as the mechanism operation of "Prolonged medical starvation" from point of view of modern scientific data, and as well as the mechanisms of interaction "Prolonged medical starvation" with considerably decrease dosage of cytotoxic drugs.

\section{The distinctions of mechanisms cellular cycle for maintenance stability IE and IM between an able- bodied tissue/cells and cancer tissue/cells}

Common mechanism of maintenance stability of IE and IM as stationary state in norm as well as quasi-stationary states in pathology is divided into three levels of regulative mechanism: highest level regulation, high level regulation and low level regulation [6-8] (Figure 2). There are mutual influencing mechanisms maintenance stability between an organism and cells of an organism which extend also as between all cells of an organism due their capacitors operations as well as between cells and their surrounding medium due to cellular capacitors operations for maintenance stability of IM and an IE of an organism and cells, displaying as immune defensive system [9]
(Figure 2). Just the mechanism of maintenance stability basophilic chemical potential of cytoplasm $\left(\mu_{\text {cytopl }}\right)$ of each cell displays the balance of mutual influences between moderately oscillating nDNA fragmentations/ reparations in nucleus and conformably moderately oscillating mtDNA fusion/ fission in mitochondria, which influence on nuclear capacitors and mitochondrial capacitors conformably [10] (Figure 2). The interactions between the related resonance waves of the nuclear capacitors and the mitochondrial capacitors create the remote reaction for maintenance of stable balance catabolic and anabolic processes in cytoplasm which induces stable basophilic chemical potential of cytoplasm $\left(u_{\text {cytopl }}\right)$ defining stable IE and IM of cytoplasm in normal quiescent $\mathrm{G}_{0}$ phase of cellular cycle [10] (Figure 3). Just oscillating changes of these resisted processes, which occur both in cells and in tissues, are mutual subjected to the central regulation of an organism promoting maintenance stability of IE [stability temperature $36^{\circ} \mathrm{C}-36.9^{\circ} \mathrm{C}$ by which all enzymes operate] and IM [constant concentration substances in blood and in neurolymph] both in an organism and in cells of an organism in norm [10] (Figure 3). Also moderate oscillating shifts of balance anabolic endergonic and catabolic exergonic processes into anabolic pathway and into catabolic pathway occur in low level of regulatory mechanism for maintenance stability stationary state an organism.

The excessive shift of the balance anabolic endergonic and catabolic exergonic processes into anabolic endergonic processes causes quasi-stationary state in cancer tissue creating the negative fluctuation of entropy according to Glansdorff and Prigogine theory $(-\Delta \times \beta)$. Such transmutation normal metabolic processes maintaining balance catabolic \& anabolic processes into excessive anabolic processes leads to change metabolic processes causing excessive irrepressible proliferations via expression nuclear activity exerting cellular cycle into G1/S phases of biosynthesis substances (proteins, lipids, carbohydrates etc.) transiting into G2/M phases of cells divisions. Thus quasi-stationary state in cancer tissue is characterized by formation Warburg effect, which causes excessive proliferative processes, irrepressible cancer growth, unhealed cancer wounds, metastasis and cancer cells apoptosis resistance [3]. Cancer mitochondria DNA produce abundance quantity reactive oxygen species (ROS) in comparison with moderate quantity ROS production in normal mitochondria DNA [12-14]. Just it is known that ROS induces superoxide $\left[\mathrm{O}_{2} *\right]$, and superoxide anion is subjected to dismutation by manganese superoxide dismutase (MnSOD) and copper, zinc superoxide dismutase ( $\mathrm{Cu}, \mathrm{ZnSOD}$ ) converting into hydrogen peroxide $\left[\mathrm{H}_{2} \mathrm{O}_{2}\right.$ ] [12]. Complex $\mathrm{ROS} / \mathrm{H}_{2} \mathrm{O}_{2}$ generates superoxide $\left[\mathrm{O}_{2}{ }^{*}\right]$ inducing free radicals $(" \mathrm{OH})$ [12]. Free radicals $\left({ }^{*} \mathrm{OH}\right)$ react on nucleus DNA and induce process replication via realizing of 2nDNA in nucleus [10]:

\footnotetext{
"OH $+\mathrm{H}_{2}$-nDNA-DNA --> $\mathrm{H}_{2} \mathrm{O}+\mathrm{H}^{\prime}$-nDNA-DNA;

$\mathrm{O}^{*}+2 \mathrm{H}_{2} \mathrm{O}-->2 \mathrm{H}^{\cdot}+2 \mathrm{OH}^{-}$;

$2 \mathrm{H}^{\cdot}-\mathrm{nDNA}-\mathrm{DNA}+2 \mathrm{H}^{\cdot}-\mathrm{-}>\mathrm{2nDNA}-\mathrm{H}^{\cdot}+2 \mathrm{nDNA}-\mathrm{H}^{\prime}$;

2 nDNA-H' $+2 * \mathrm{OH}-->2 n D N A+2 \mathrm{H}_{2} \mathrm{O}$
} 
The greatacceleration of cellularcycle, induced by oncogene, is occurred in cancer cells. The part of complex ROS/ $\mathrm{H}_{2} \mathrm{O}_{2}$ in mitochondria is neutralized by glutathione peroxidise (GPX) and phospholipid hydroperoxide glutathione peroxidise (PHGPX) in G1 phase oncologic cellular cycle [10, 13-18]. The produced excessive abundance of complex ROS/ $\mathrm{H}_{2} \mathrm{O}_{2}$ in $\mathrm{G} 2$ phases oncologic cellular cycle pass through mitochondrial membranes and cytoplasm into nucleus and generates excessive abundance of superoxide $\left[\mathrm{O}_{2}{ }^{*}\right]$ inducing excessive abundance of free radicals $\left({ }^{*} \mathrm{OH}\right)$. The excessive free radicals influence on nuclear DNA inducing processes of permanent DNA replications which also cause neutralization of abundance complex $\mathrm{ROS} / \mathrm{H}_{2} \mathrm{O}_{2}$ / Free radicals (equations above) [10]. All processes of mitochondrial biogenesis are advanced due to nitric oxide both in normal cellular cycle development and in oncologic cellular cycle development exhibiting partial transfer from catabolic processes into excessive anabolic processes for cancer cells survival as apoptosis resistance that cause mechanism aerobic glycolysis of Warburg effect $[10,11$, $12,18-22]$.

Short description and explanation of preliminary preparation for following use cancer treatment via "Prolonged medical Starvation during 42 - 45 days" [4, 5, 10]

Prolonged medical starvation should be supplemented by considerably decreased dosage cytotoxic substances! "Prolonged medical starvation during 42-45 days" should be defended with the support of herb extracts [sage, hawthorn, horsetail, (stinging-) nettle, ninety-knot, hypericum, ergot, St. John's wort, etc.] providing with cytotoxic activity of red cranesbill (Geranium robertianum) and used abundant liquid drink including water up to 1.5-2.0 liter per day. The herbal extracts should be filtered through a triple gauze layer in order that any fibre mustn't remain in the extract. The herbal extracts fill the organism during "Prologed medical starvation 42-45 days" treatment with necessary microelements and vitamins, especially folic acid, that is necessary for hemopoiesis and decreases also acidification in the blood of the organism by "Prolonged medical starvation". During the "Prolonged medical starvation" it's necessary to look after the common health state of the person and especially state of gastrointestinal tract that it occurs the bowels open/ timely evacuation of excrements/, that there will not be constipation/ retention of feces. The disturbance of gastrointestinal activity should be healed with vegetable laxatives, activated charcoal, medicaments and use an enema if it's necessary. The starvation leaving should been taken place during 7 days with gradual addition of products: juices, then watery decoctions and gels, then vegetable pulps, then baked fruits and vegetables, then liquid kasha (dish of cooked grain), then mashed potatoes, then pair of cutlets- and up to the usual nutrition. The diet shouldn't be salted during leaving starvation.

Considering successful great experience of treatment oncologic diseases, it should been familiarized with the Rudolf Breuss recommendations for using extracts of herbs during "Prolonged medical starvation 42-45 days" [1, 2]

Taking into account the great experience of folk healer R. Breuss in treatment of various cancer diseases, there are R. Breuss recommendations concerning using extracts of herbs $[1,2]$. There is retransmission of these recommendations in the article: It should be used three teas for all types of cancers: sage tea, kidney tea and cranesbill tea.

Sage tea includes sage (Salvia officinalis), St. John's wort (Hypericum perforatum), peppermint (Mentha piperata) and balm (Melissa officinalis): One teaspoon or maximum two teaspoons of sage should be poured into boiling water and boiled only three Min. Then sage should be eliminated via filtering through a triple gauze layer in order that any fibre mustn't remain in the extract. St. John's wort (Hypericum perforatum), peppermint (Mentha piperata) and balm (Melissa officinalis) should be poured into this hot extract and drawn ten Min more and then filtered through a triple gauze layer. Thus sage tea is obtained as the extract of these herbs.

Kidney tea includes horsetail (Equisetum arvense) - 15 grams, stinging nettle (Urtica dioica) - 10 grams, knotgrass (Polygonum aviculare) - 8 grams and St. John's wort (Hypericum perforatum) - 6 grams. The pinch of these herbs mixture should be drawn ten Min in a cup of hot water. These herbs mixture should be filtered through a triple gauze layer. The hard rest of herbs mixture must be poured over with hot water and boiled ten Min. Then herbs mixture should be again filtered and both these filtered liquids should be mixed. The explanation of such mode of preparation kidney tea is that there are the required substances in the filtered liquid of these herbs mixture which should not be boiled because of these substances decay. However there is silicic acid in the hard rest of herbs mixture which can be received by ten Min boiling. R. Breuss notes that kidney tea must be taken only for the first three weeks. May be R. Breuss thought that kidney tea is meant for training kidneys of an organism to "Prolonged medical treatment 42-45 days".

Cranesbill tea includes red cranesbill (Geranium robertianum). The pinch of the red cranesbill (Geranium robertianum) should be drawn ten Min in a cup of hot water and filtered through a triple gauze layer. Only one half cup of cold cranesbill tea should be ingested per day. R. Breuss notes that red cranesbill (Geranium robertianum) contains the small quantity of radium. Thus the new method of treatment is integrated with up-todate methods of treatment via using the small quantity of radium as the cytotoxic remedy in condition of the long medical starvation.

Besides R. Breuss offers to use during treatment via 42 days starvation the vegetable juice mixture which consist of $55 \%$ red beet root - 300 grams, $20 \%$ carrots -100 grams, $20 \%$ celery root, 100 grams, $3 \%$ raw potato -10 to 30 grams options, and $2 \%$ black radish - 10 to 30 grams. He let know that the vegetable juice mixture should not be drunk more than half a liter per day. It should be mixed all these vegetables in a juicier and then strain to get rid of any sediment. Before starting the cure it can try one quarter liter of juice per day with normal meal taking 
this juice by the spoonful. Also R. Breuss notes that the potato is optional except for liver cancer where it plays an important part. As concern to content sugar in red beets, potato and carrots of vegetable juice mixture offered by R. Breuss, it should been taken only into consideration taking into account his great successful experience in treatment cancer diseases. However the author has followed also folk healer Omelchenko $A$. experience who used only extracts of herbs and did not use any vegetables in treatment of cancer disease, receiving also positive results. Unfortunately folk healer Omelchenko A. concealed the names of herbs which he used. Therefore the author has used the herbs advised by R. Breuss, but he did not use vegetables in his short successful experience of treatment cancer disease (see below).

The R. Breuss instructions are to take one half cup of cold kidney tea first thing in the morning for the purpose of habituation. Then 30 to 60 Min later take two cups warm herb tea made from St. John's wort, peppermint and lemon balm. After another 30 to 60 Min take a little vegetable juice and salivate well before swallowing. Fifteen to 30 Min later take another sip of vegetable juice. It should be taken some juice 10 to 15 times throughout the morning but only when patient feels like it. Then patient can drink warm or cold sage tea without sugar, as much as he like. At noon it should be taken one half cup of kidney tea and again before retiring at night. In the afternoon it should be taken a few sips of tea. Any liquid should be sipped and well salivated. Kidney tea should be taken only during the first three weeks of the treatment. Also R. Breuss insists on urgent request to drink one cup of cold cranesbill tea (Geranium robertianum) per day throughout the time of therapy. Besides R. Breuss recommends for cancer treatment of the bones and lungs, prepare a tea of plantain (Plantago major), icelandic moss, lungwort, ground ivy and mullein.

Footnote: Cranesbill tea (Geranium robertianum) contains cytotoxic substances (see below).

The contraindications to the use of "Prolonged medical starvation 42 - 45 days"

The irreparable cancerous damage of IE and IM an organism are the basis of the contraindications to the use of the offered method cancer treatment. So the generalized excessive shift of balance anabolic and catabolic processes into the huge anabolic processes via abundant metastasis suppresses catabolic processes critically. Just catabolic processes generate energy and dissipate energy into environment promoting stability energy $\left[36.6^{\circ} \mathrm{C}-37.3^{\circ} \mathrm{C}\right]$ for maintenance stability IE an organism, i.e. catabolic processes contribute to survival of an organism. Thus aggressive processes of metastatic disease create apoptosis resistance in cancer cells and enhance apoptotic processes in an organism. These destructive processes in the organism lead to damage of IE of the organism, i.e. the symptoms which are the contraindication to the use prolonged medical starvation [42-45 days].
Thus there are the contraindications to the use of the offered method cancer treatment: Cachexia is the first cause of contraindication to the use of the offered method cancer treatment, because progressive loss of weight indicates the full downfall of energy forces of the organism and also full downfall of its defensive system, promoting irrepressible development of cancer tumor. Considering such physical state of the organism, the prolonged medical starvation can not deprive cancer tissue of substances for cancer metabolism.

Full collapse of cancer sick patient is the second cause of contraindication to the use of the offered method cancer treatment. This contraindication shows symptoms both unstable equilibrium state of body and helplessness of the person. Full frailty also indicates the full downfall of energy forces of the organism and also full downfall of its defensive system, promoting irrepressible development of cancer tumor. Considering such physical state of the organism, the prolonged medical starvation cannot deprive cancer tissue of substances for cancer metabolism.

Cancerous intoxication due to the decomposition of cancerous necrotic mass is the third cause of contraindication to the use of the offered method cancer treatment. Cancerous intoxication becomes apparent as sickness with uncontrollable vomiting, anorexia and bad physical state of the organism. Cancerous intoxication indicates irrepressible development of cancer tumor owing to the full downfall of energy forces of the organism and also full downfall of its defensive system. Considering such physical state of the organism, the prolonged medical starvation cannot deprive cancer tissue of substances for cancer metabolism.

Dangerous metastasis for life is the fourth cause of contraindication to the use of the offered method cancer treatment. There are such dangerous for life metastasis: metastasis in brain, metastasis in spinal marrow, vast metastasis in liver causing huge liver and so on.

The tumors, which create emergency needing to urgent medical /especial surgical/ help, should not be treated with prolonged medical starvation of the new method cancer treatment before rendering first aid of urgent medical surgical help. There are such emergency situations: large bowel obstruction [ileus], urinary obstruction, biliary tract obstruction, airways obstruction, duodenal obstruction, small bowel obstruction, and tumor location in vital center or in vital organs which can be ablated by surgical methods. It is the fifth cause of contraindication to the use of the offered method cancer treatment.

\section{The results of practical observations, regarding "Prolonged medical starvation"}

Borrowing from the folk healers Omelchenko A and Breuss R. the method "Prolonged medical starvation" for cancer treatment, the author was convinced of the efficiency of this method treatment by the meetings with more than 
100 cured patients during 2 years, who were treated by folk healer Omelchenko. The studies of the "Medical cards" some of these cured persons have convinced the author that the folk healer Omelchenko A. has treated the incurable ill men and has received positive results. The patients have obtained the initial diagnoses in oncologic hospitals of official medical practice, but further they were treated by the folk healer Omelchenko. Unfortunately the folk healer Omelchenko A. had only the apparatus for ultrasound investigations. Therefore the results of treatment were based only on the data of the ultrasound investigations and the clinical observations.

Thus the patient Gr. was diagnosed with cancer kidney $Y$ degree, clinical stage IY, with metastases in the abdomen cavity and liver. This diagnosis was diagnosed in the "Kiev Oncologic dispensary" and was confirmed by the folk healer Omelchenko A. via the clinical investigations and the ultrasound investigations. After treatment the ultrasound investigations and clinical investigations showed disappearance the dense focus of liver. Also the clinical investigations showed disappearance of abdomen metastases. The patient has felt fine and has gone to his work.

The other patient Mo. was diagnosed with cancer ventricle $\mathrm{Y}$ degree, clinical stage IY, with metastases in the abdomen cavity and liver. After treatment the ultrasound investigations and clinical investigations showed disappearance the dense focus of liver. Also the clinical investigations showed disappearance of abdomen metastases.

Also we have met with the some other cured patients with various locations of cancer tumors, who were treated by the folk healer Omelchenko A., although these patients were not incurable cancer patients from the point of view of the modern medicine. Therefore these patients could not show the initial diagnoses from the oncologic hospitals.

Austrian folk healer Breuss R. used for treatment “Prolonged medical starvation during 42 days" and described a lot of the patients with various locations of cancer tumor and with various locations of metastases, who were cured by this method of treatment [1, 2]. The data, described by the austrian folk healer Breuss R., concerning results of this method treatment were also convinced us in efficiency of this method treatment $[1,2]$.

The author was also convinced on own experience the efficiency of treatment the ill man with the incurable cancer stage. Here is own experience in the treatment of the patient with the incurable cancer disease. At February 1998 the patient Ch. has been operated for cancer of the left kidney (the diseased kidney was resected).

On 24 February 1999, it was detected by the X-ray inspection in the "Kiev Oncologic dispensary" and diagnosed metastatic cancer, the intermediate bronchus of the right lung, IY degree, clinical stage IY. After detail examines the patient Ch. has been discharged from Oncologic dispensary how the incurable patient. Then the patient $\mathrm{Ch}$. and relatives of the patient have agreed to receive prolonged medical starvation treatment with the examinations in the "Kiev Institute of Roentgenology, Radiology and Oncology".

On 24 February 1999, the patient was examined in the "Kiev Institute of Roentgenology, Radiology and Oncology" before prolonged medical starvation treatment. Histological examination of sputum from a bronchus, the fragment of necrotic masses of a disintegrating malignant tumour and a clump of polygonal cells of a tumor of not forming complexes and frames is detected in the stuff. The histological pattern mismatches a lung carcinoma but specifies in the metastasis from the tumor of kidney (hypernephroma). The result of X-ray inspection was: Metastatic cancer the intermediate bronchus of the right lung IY degree, clinical stage IY. The diagnosis is remained the same how Oncologic dispensary. The treatment was occurred from 01 March 1999.

The examination after prolonged medical treatment: On $X$-ray patterns there are not found pathological changes in the lungs. The medical examinations of the patient during three years (catamnesis) and the examination in 2002 at the "Kiev Institute of Roentgenology, Radiology and Oncology" show that the patient was cured.

\section{The mechanism operation "Prolonged medical starvation 42-45 days" in cancer therapy}

Prolonged medical starvation as the new approach to cancer therapy activates catabolic processes in an organism for maintenance stable temperature $36.6^{\circ} \mathrm{C}-37.3^{\circ} \mathrm{C}$ by which all enzymes operate. Cancer tumor is situated inside the human organism using the organism as environment, and obtains the substances for its metabolism from depot of an organism (fat depots, carbohydrate depots etc.) [4, 5]. Also an organism obtains substances for its metabolism from depots of an organism in condition of treatment by "Prolonged medical starvation (during 42-45 days)". Besides, the treatment by "Prolonged medical starvation (during 42-45 days)" causes considerable decrease almost of all depots (especially fat depots) of an organism. Therefore this method treatment leads to the competition between cancer tissue and an organism for the use of remained decreased depot to maintain the normal temperature $\left(36.0^{\circ} \mathrm{C}-37.5^{\circ} \mathrm{C}\right)$ by which the all enzymes operate. The aerobic exothermic oxidation, using for maintenance stability of the normal temperature $\left(36^{\circ} \mathrm{C}-37.5^{\circ} \mathrm{C}\right)$ as IE of an organism, generates the greatest quantity of calories promoting suppression of anabolic endergonic processes in the condition of the treatment by "Prolonged medical starvation (during 42-45 days)" as in the organism as well as in cancer tissue. Thus this competition between the organism and the cancer must lead to the win for most strong one. But the protective forces of the organism become stronger due to support with herbal extracts, delivering vitamins and microelements into the organism. Also increase of fat metabolism from fat depot leads to augmentation glutathione peroxide (GPX) and phospholipid hydroperoxide glutathione peroxidise (PHGPX) in all 
cells of an organism and contributes to neutralization of redundant ROS in G1/S phases cellular cycle of cancer cells [10]. Thus "Prolonged medical starvation" promote suppression DNA replication due to ROS/free radicals neutralization in G1/S phases cellular cycle before nDNA replication in G2/M phases cellular cycle, as in an organism and as well as in cancer cells. Firstly, the decrease any substances (including glucose) as in cytoplasm as well as in blood serum cannot influence on nDNA replication, and secondly, "Prolonged medical starvation" cannot decrease concentration substance both in cytoplasm and in blood due to such mechanisms as gluconeogenesis and the similar others maintaining stability IM both in cytoplasm and in blood as well as in neurolymph (stable concentration substances in cytoplasm, blood and neurolymph). The suppression nucleus DNA replication causes suppression of cellular cycle in cancer cells and cessation of irrepressible proliferative processes with irrepressible cancer growth. In addition, at the beginning of fasting (at 5-7 and 12-14 days) there arises the short acidophilic reaction with formation of ketonic bodies as the result of intensive fat oxidation from fat depots of the organism. As the result of $\beta$-oxidation of fat acids many Acetyl ions [ $\left.\mathrm{CH}_{3} \mathrm{CHO}-\right]$ are produced. The great quantity of Acetyl ions increases quantity Acetyl-CoA for catabolic processes that promote partial elimination of overload "the nodal point of bifurcation of anabolic and catabolic processes" (NPBac), causing elimination of Warburg effect. Acetyl-CoA consumption by the increased oxidative phase of metabolism (Krebs cycle) causes the decrease of excess lactic acids which accumulate energy for anabolic processes, especially needing for cancer tissue [3-5]. The cessation of the huge consumption energy and Acetyl-CoA for anabolic processes in cancer metabolism ruins the mechanism of Warburg effect and restores as balance catabolic and anabolic processes as well as the mechanism of Pasteur effect /incompatibility of aerobic processes with glycolysis [3-5]. The cessation of the overload of "nodal point of bifurcation anabolic and catabolic processes" (NPBac) promotes the normal excretion of synthesized high-molecular substances via oxidative processes and ruins the huge excessive Alternative excretion which causes metastasis and nonhealing tumor ulcers formation [3-5]. The elimination of the overload of "nodal point of bifurcation anabolic and catabolic processes" (NPBac) restores "contact inhibition of cell propagation" how in the normal tissue and ruins the irrepressible tumor growth [3-5]. Thereby Warburg effect, characterizing by aerobic glycolysis, is destroyed because of expression aerobic catabolic processes and decrease anaerobic processes of glycolysis. Destruction of Warburg effect violates cancer metabolism and contributes to normal metabolism with Pasteur effect.

Thus basic phenomena of the cancer metabolism are inhibited: a) Mechanism of "Warburg effect". b) Biochemical and biophysical mechanisms of metastases and non-healing tumor ulcers formation. c) The phenomenon of "absence inhibition of contact cell propagation in the metabolism of malignant tumor" and as well as irrepressible tumor growth.

\section{Benefits of the use "Prolonged medical starvation" in cancer therapy}

"Prolonged medical starvation" contributes to depression of cancer tumor metabolism that helps for efficient anticancer therapy with decreased dosage of cytotoxic drugs. Such approach to anticancer chemotherapy prevents damage IE and IM both an organism and cells of an organism, preventing damage of immune and hormonal systems as the links of defensive mechanism in regulative system of an organism. Prevention damage of immune and hormonal systems as the links of system stability IE and IM an organism prevents recurrence of cancer disease after long anticancer chemotherapy and resistance to anticancer drugs in process of intensive anticancer chemotherapy with great dosage of cytotoxic drugs.

Highlight: The huge anabolic processes with huge consumption of energy and Acetyl-CoA are characteristic for cancer tissue. These processes suppress the catabolic exergonic processes in cancer tissue retaining only the rest catabolic exergonic processes for cancer cells survival. Just "Prolonged medical starvation" induces shift balance catabolic and anabolic processes of organism's tissue metabolism into expression of catabolic exergonic processes for maintenance stability of IE of the organism (the stable temperature an organism $36.0^{\circ} \mathrm{C}-37.2^{\circ} \mathrm{C}$ and the other biophysical parameters as $\mathrm{PH}$, osmotic pressure etc. in blood and in neurolymph), that suppress anabolic endergonic processes characteristic for cancer tissue leading to tumor depression [3, 4]. "Prolonged medical starvation" promote suppression DNA replication, due to ROS/ free radicals neutralization in G1/S phases cellular cycle before nDNA replication in G2/M phases cellular cycle, as in an organism and as well as in cancer cells. The suppression nucleus DNA replication causes suppression of cellular cycle in cancer cells and cessation of irrepressible proliferative processes with irrepressible cancer growth. The use light cytotoxic herbal extract in condition of prolonged medical starvation leads to transition of tumor depression into damage of tumor metabolism and to cure of the patient.

\section{The mechanism operation of the extracts of herbs in new method of cancer treatment}

Considering the above described mechanism operation of the treatment by "Prolonged medical starvation during 42-45 days", this method of cancer treatment leads to depression of development tumor as the result of damage the main mechanisms of cancer tumor metabolism. The crucial role of maintenance IE stability of the organism in condition of prolonged medical starvation appertains to the all tendered extracts of herbs as well as to the vegetable juice mixture, which deliver to an organism necessary microelements and vitamins, especially folic acid, that is necessary for hemopoiesis, and decreases also acidification in the blood of the organism. It must especial pay attention on the extract of red cranesbill (Geranium robertianum) which contains significant amounts of vitamins $A, B$ and $C$ as well as such minerals: calcium, potassium, magnesium, iron, phosphorus, germanium, 
according to Shipard Isabell [23]. Besides, R. Breuss notes that red cranesbill (Geranium robertianum) contains the small quantity of radium [1, 2]. Also Shipard Isabell [23] notes that Geranium (or Herb Robert) has wide range of clinical applications as remedy with such properties: antibiotic and antiviral properties, sedative property, tonic, astringent, diuretic, digestive and antioxidant. It should be paid attention on especial importance that Geranium (or Herb Robert) is a source of germanium [23] and radium $[1,2]$. Helfer $M$. et al. show effect of Geranium on HIV-1 as antiviral remedy [24]. Also taking into account that red cranesbill (Geranium robertianum) has antibiotic and antiviral properties $[23,24]$, it can assume that red cranesbill (Geranium robertianum) has also light cytotoxic property as concern radium which cytotoxic properties do not raise the doubts. Thus it can assume that red cranesbill (Geranium robertianum) causes light cytotoxic property on depressed malignant tumor in condition of prolonged starvation during 42-45 days, promoting cancer disease treatment and cure of patient. Such light cytotoxic property cannot make negative influence on immune and hormonal systems of an organism essentially as opposed to chemotherapy with great dosage cytotoxic drugs.

Advantage action of small dosage cytotoxic drugs on depressed cancer metabolism for prevention cancer disease recurrence and drugs resistance to the cytotoxic effects in offered method cancer treatment over action of great dosage cytotoxic drugs in modern methods cancer therapy

Unlike the modern methods of cancer disease treatment the offered method of cancer disease treatment via "Prolonged medical starvation with very small dosage cytotoxic drugs" does not intrude into immune and hormonal systems and does not violate the stability as an organism IE and IM as well as cells of an organism causing destruction of cancer tumor. Therefore the offered method of cancer disease treatment via "Prolonged medical starvation with very small dosage cytotoxic drugs" does not result in appearance of epigenetic changes and genes amplification causing drugs resistance to the cytotoxic effects and does exert cancer disease recurrence after some medical remissions which occur as the result of damage both immune and hormonal systems by great dosage cytotoxic drugs using in up-todate methods cancer therapy. However the combination of the described offered method treatment with up-todate methods treatment should be approved in clinical condition as clinical trials.

Highlight: The inhibition of proliferative anabolic properties of cancer cells is achieved via the rearrangement of cancer metabolism from anabolic pathway into catabolic pathway in the treatment via "Prolonged medical starvation". Thus this method cancer treatment puts cancer metabolism into state of depression and preserves proliferative anabolic functions of immune and hormonal system and as well as IE and IM an organism. Then the light cytotoxic effect of small dosage of anticancer drugs in condition of cancer depression does not exert mechanism of drugs resistance to the cytotoxic effects and recurrence cancer disease after remission of cancer disease, but lead to destruction cancer metabolism and recovery of ill man.

\section{Discussion the benefit of combination "Prolonged medical starvation" with the decreased dosage cytotoxic drugs for efficient cancer therapy}

Critical reviews of mechanisms drugs resistance to the cytotoxic effects due to the intensive course of patients treatment with the great dosage of cytotoxic therapy.

Mechanism of cordycepin (3'-deoxyadenosine) cytotoxic effect Describing mechanism of cordycepin (3'-deoxyadenosine) cytotoxic effect, Imesch P, et al. [25] found that cordycepin cytotoxic effect via study both transcription processes as an inhibitor of poly(A) polymerase (PAP) and DNA replication focused on MLH1 [one of the five DNA mismatch repair (MMR) proteins], i.e. processes which operate in G2/M phases of cellular cycle. Also they noted that cells with defective MMR function showed resistance to certain anticancer drugs. So Imesch P, et al. [25] found that MLH1deficient tumor cells exhibited reduced susceptibility to apoptosis upon treatment with cordycepin, as compared to MLH1-proficient tumor cells.

Also studying cytotoxic effect of lipoplatin (a novel liposomal cisplatin exhibiting highly effective action against cancers), Fedier A, et al. [26] revealed that MLH1-deficient tumor cells were less susceptible to apoptosis than MLH1-proficient tumor cells. However they noted that MLH1-deficient tumor cells showed the same sensitivity to lipoxal (a novel liposomal drug - oxaliplatin) as well as MLH1-proficient tumor cells. Besides, Sergent C, et al. [27] have supposed that high level resistance of human colon cancer cells to high doses of cisplatin and oxaliplatin does not seem to be related to acquired defects in the DNA MMR proteins.

a) Mechanisms of experiments outcomes: Cancer tissue metabolism is characterized by huge anabolic processes, promoting irrepressible proliferative processes via advance $\mathrm{G} 1 / \mathrm{S}$ phases of cellular cycle $[3,8,10]$. Development cellular cycle requires mismatch repair (MMR) proteins (enzymes) for repairs of base-base mismatch, that occur due to DNA replication in G2/M phases cellular cycle as in MLH1proficient tumor cells as well as in MLH1-deficient tumor cells. Therefore it can be such case by MLH1-deficient tumor cells that the function repair of DNA mismatch is distributed as among the all mismatch repair (MMR) proteins (nine genes of MMR function) as well as among the main five genes of MMR function (MLH1, PMS1, PMS2, $\mathrm{MSH} 2$, and MSH6). Thus the operative area of the cytotoxic drugs become wider and their cytotoxic effects are divided among these proteins decreasing dosage of cytotoxic drugs on each protein and also decreasing susceptibility to apoptosis upon treatment with these drugs. Only strong cytotoxic drugs with cytotoxic influence on both anabolic processes and catabolic processes in both G1/S and G2/M phases cellular cycle show the similar cytotoxic effects in both MLH1-deficient tumor cells and MLH1-proficient tumor cells. Besides there is the other mechanism, remote cellular reactions between cells and cytotoxic drug, which are carried out by system of cellular capacitors, precede cellular contact reactions with substances of cytotoxic drugs [9]. Remote cellular reactions set cells to accept the substance of cytotoxic drug. Also remote reactions cells 
cause attraction between cells and substance of cytotoxic drug which can be weaker in MLH1-deficient tumor cells than in MLH1-proficient tumor cells due to violation interactions between nDNA and mtDNA, resulting in violation operations of nucleus capacitors - mitochondria capacitors link that influence on stability basophilic chemical potential cytoplasm and on cellular inner membrane charge of cellular capacitors $[9,10]$. Therefore the remote cellular reaction between MLH1-proficient tumor cells and cytotoxic drug can be more absolute than with MLH1deficient tumor cells. Hence the acting of cytotoxic drug in contact reaction with MLH1-proficient tumor cells can be greater than with MLH1-deficient tumor cells. Thus MLH1deficient tumor cells can be less susceptible to cytotoxic drug and to apoptosis than MLH1-proficient tumor cells, i.e. drug resistance occurs in MLH1-deficient tumor cells. On the other hand, the interactions between remote cellular reactions and contact cellular reactions show the following sequence of the reactions between cells and cytotoxic drug: Cellular capacitors react on substance of cytotoxic drug and promote reaction of cells on cytotoxic drug [9]. Simultaneously mutual interactions between cellular capacitors and link of nucleus capacitors-mitochondria capacitors promote rearrangement mismatch repair (MMR) function in nucleus, connecting with molecular structure of cytotoxic drug substance. Such rearrangement mismatch repair (MMR) function in nucleus can lead to such case that the operative area of the cytotoxic drug become wider due to distribution of the function reparations of DNA mismatch as among the all mismatch repair (MMR) proteins (nine genes of MMR function) as well as among the main five genes of MMR function (MLH1, PMS1, PMS2, $\mathrm{MSH} 2$, and MSH6) in MLH1-deficient tumor cells. Thus the drug cytotoxic effect in MLH1-deficient tumor cells is divided among the mismatch repair (MMR) proteins decreasing dosage of cytotoxic drug on each protein and also decreasing susceptibility to apoptosis upon treatment with this drug.

b) Footnote: The advantage of the combination very light cytotoxic effect of the small dosages of cytotoxic drugs [cordycepin, cisplatin, lipoplatin, lipoxal (oxaliplatin) etc.] with the offered method cancer disease treatment is that the offered method cancer disease treatment leads to depression of cancer tumor metabolism due to suppression anabolic processes, and the cytotoxic effects of the small doses drugs become stronger over the suppressed anabolic processes of cancer tumor as compared with great dosage cytotoxic drugs in the state of cancer cells activity in modern methods cancer therapy. Just the great dosages of cytotoxic drugs show the similar cytotoxic effects as on hormonal cells and immune cells of an organism's defensive function as well as on both MLH1deficient tumor cells and MLH1-proficient tumor cells, i.e. they interfere in IM an organism suppressing defensive function of it.

The investigations influences of PI3K/AKt cascade inhibitors on efficiency of cytotoxic effects caused by medical drugs and focused on MLH1-deficient tumor cells or on MLH1-proficient tumor cells

Ohta T. et al. [28] and Stathopoulos GP et al. [29] note that the PI3K/Akt cascade displays an important role in the resistance of ovarian cancer cells to cisplatin.
Fedier A, et al. [30] investigated interdependence between cytotoxic effect of lipoplatin and AKt inhibitor LY294005. Their outcomes showed that LY294005 /inhibitor of AKt/ decreases the efficacy of cisplatin, lipoplatin, oxaliplatin as well as lipoxal in human colorectal adenocarcinoma, but, unlike these drugs, LY294005 increases the efficacy of docetaxel and does not affect the efficacy of 6-thioguanine. Fedier A, et al. [30] prolonged the study of mechanisms AKt inhibition by LY294005 investigating the function of DNA mismatch repair (MMR) in MLH1-deficient tumor cells and in MLH1-proficient tumor cells. The results of researches show that the influence of LY294005, resulting in decreases efficiency with cisplatin and lipoplatin, is significantly higher in the MLH1-deficient than in the MLH1- proficient, but nearly similar efficiency with oxaliplatin and lipoxal. Moreover LY294005 in the MLH1-deficient increases sensitivity with docetaxel and decrease sensitivity with platinum compounds drugs that cannot be associated with the concomitant deletion of the phospho-Aktser473 level. On the contrary, analogous changes in drug sensitivity were observed with the PI3-kinase inhibitor LY294002, but these changes were associated with complete deletion of phospho-Aktser473. Fedier A, et al. [30] supposes a possible relationship between MMR-mediated with cisplatinum DNA damage and action AKt, e.g. a common target for both pathways. Simultaneously Fedier A, et al. [30] express desire that the possible new property of Akt in making influences on drug sensitivity may also be proposed.

a) Mechanisms of experiments outcomes: AKt stimulates glycolysis activating hexokinase 2 (HK-2), i.e. AKt promotes the first an irreversible step in glycolysis, according to Elstrom RL, et al. [31] and Gottlob K, et al. [32]. Besides, Akt promotes growth factor, according to data Plas DR, et al. [33, 34]. Just Akt pathway leads to produce Acetyl$\mathrm{CoA}$ via stimulating glycolysis. Thus AKt stimulates both anabolic endergonic pathway and catabolic exergonic pathway which are formed from Acetyl-CoA of "nodal point bifurcation of anabolic endergonic processes and catabolic exergonic processes"/ NPBab/ [3, 4] (Figure 1). Cisplatin and lipoplatin damage cellular nucleus, violating nuclear basis of anabolic processes. However they do not put obstacles in the AKt operation of inducing catabolic exergonic processes and anabolic endergonic processes via glycolysis in cytoplasm. Really it is possibility that LY294005/ inhibitor of AKt/ decreases the efficacy of cisplatin, lipoplatin, oxaliplatin due to relationship between MMRmediated with cisplatinum DNA damage and action PI3K/ AKt inhibitors. Just both AKt inhibitor LY294005 and PI3kinase inhibitor LY294002 violate glycolysis in cytoplasm that cause violation of mismatch repair (MMR) function due to lack of Acetyl-CoA, violating in cytoplasm both anabolic function (connected with nucleus function) and catabolic function (connected with mitochondrial oxidative function) and converting cells into MLH1-deficient tumor cells. Thus mechanisms operations of these drugs show that they more decrease activity in MLH1-deficient tumor cells than in MLH1-proficient tumor cells, because the function repair of DNA mismatch is distributed in MLH1-deficient tumor 
cells as among the all mismatch repair (MMR) proteins (nine genes with MMR function) as well as among the main five genes with MMR function (MLH1, PMS1, PMS2, MSH2, and MSH6). Therefore the operative area of these cytotoxic drugs become wider and their cytotoxic effects are divided among these proteins decreasing dosage of cytotoxic drug on each protein and also decreasing susceptibility to apoptosis upon treatment with these drugs in MLH1deficient tumor cells. Docetaxel exhibits also strong antimitotic chemotherapy, i.e. Docetaxel violates both nuclear function and mitochondrial function. Therefore LY294005 in the MLH1-deficient increases sensitivity with docetaxel and decrease sensitivity with platinum compounds.

b) Footnote: Taking into account these researches it can affirm that the advantage of the combination very light cytotoxic effects of the small dosages of cytotoxic drugs [cisplatin, lipoplatin, docetaxel, etc.] with the offered method cancer disease treatment is similar to above described mechanism suppression depressed cancer metabolism with small dosage of cytotoxic drugs over suppression active cancer metabolism with great dosage cytotoxic drugs in up-to-date methods of cancer therapy which cause interference in IM an organism suppressing defensive function of an organism.

Influence of radicicol, heat shock protein 90 (HSP90) inhibitor, on sensitivity to cisplatin in presence of MLH1 protein

Fedier A, et al. [35] have found that the influence of radicicol, heat shock protein 90 (HSP90) inhibitor, on sensitivity to cisplatin in presence of MLH1 protein. Their data demonstrated that radicicol increased the sensitivity to cisplatin and to oxaliplatin in both MLH1-proficient cells and MLH1-deficient cells, but considerably higher in MLH1proficient cells than in MLH1-deficient cells. Considering data that radicicol is a novel specific inhibitor for heat shock protein 90 (HSP90), they have supposed a possible functional relationship between HSP9O and MLH1, where HSP90 might affect the function of MLH1 in a way that this leads to the counter-regulation of cytotoxic pathways initiated by MMR as a consequence of the presence of DNA damage introduced by cisplatin.

a) Mechanisms of experiments outcomes: Here is the mechanism decrease generating energy for maintenance stability of IE an organism (stable temperature $36.6^{\circ} \mathrm{C}-$ $37.2^{\circ} \mathrm{C}$ by which all enzymes operate) in condition of high temperature in environment. Catabolic processes of glycolysis carry out peculiar functions, unlike the subsequent catabolic processes after "nodal point bifurcation of anabolic and catabolic pathways (NPBac)" [3]. Just catabolic processes of glycolysis generate energy. This energy is divided into anabolic and catabolic processes in "nodal point of bifurcation anabolic and catabolic processes" (NPBac), also the part of this energy is cumulated into Lactic acids for anabolic processes [3]. Thus glycolysis is the primer for both catabolic and anabolic processes. The subsequent catabolic processes, which are formed as the result bifurcation of anabolic and catabolic processes in NPBac, dissipate energy into environment for maintenance stable IE an organism, i.e. temperature $36.6^{\circ} \mathrm{C}-37^{\circ} \mathrm{C}$ by which all enzymes operate in an organism.
The stable temperature $36.6^{\circ} \mathrm{C}-37^{\circ} \mathrm{C}$ by which all enzymes operate in condition of high temperature in environment demands of suppression generating energy via inhibition glycolysis. Thus the heat shock protein 90 (HSP90) takes part as the link in mechanism inhibition of glycolysis in condition of high temperature in environment, i.e. condition of the heat shock. However the maintenance stable temperature $36.6^{\circ} \mathrm{C}-37^{\circ} \mathrm{C}$ by which all enzymes operate in normal temperature condition occurs via suppression of shock protein 90 (HSP90). It is achieved by radicicol as inhibitor heat shock protein 90 (HSP90). Therefore it is a possible consequence of counter-relationship between HSP90 and radicicol, which influences on balance anabolic and catabolic processes, i.e. on anti- and pro-proliferative pathways via suppression and expression of glycolysis: The radicicol induces pro-proliferative pathway in G2/M phases cellular cycle inhibiting HSP90 as well as MLH1. Just the replicative bypass in drug resistance mediated by loss of MMR can occur with interactions between heat shock protein 90 (HSP90) or p53 (anti-proliferative pathways) and mismatch repair (MMR) mechanisms (pro-proliferative pathways). The possible functional counter-relationship between HSP90 and mismatch repair (MMR) mechanisms reflects counter-relationship between HSP90 and radicicol. Just the suppression both catabolic and anabolic processes is characterized for HSP90. On the contrary, the function of mismatch repair (MMR) mechanisms is characterized by maintenance anabolic reparative processes for the advance of G2/M phases of cellular cycle. Just influence of radicicol repairs the mismatch repair (MMR) function. Therefore suppression of HSP90 by radicicol impels mismatch repair (MMR) function, which sensitivity to cisplatin and to oxaliplatin is increased in both MLH1-proficient cells and MLH1-deficient cells. However sensitivity to cisplatin and to oxaliplatin is considerably higher in MLH1-proficient cells than in MLH1-deficient cells, because the function reparations of DNA mismatch in MLH1-deficient cells distribute as among the all mismatch repair (MMR) proteins (nine genes with MMR function) as well as among the main five genes with MMR function (MLH1, PMS1, PMS2, $\mathrm{MSH} 2$, and MSH6). Thus the operative area of the cytotoxic property of cisplatin or oxaliplatin become wider and the cytotoxic effects are divided among the mismatch repair (MMR) proteins decreasing dosage of cisplatin cytotoxic effect on each protein and also decreasing sensitivity to cisplatin or to oxaliplatin, showing more resistance to cytotoxic drugs in MLH1-deficient tumor cells than in MLH1-proficient tumor cells.

b) Footnote: The advantage of the combination very light cytotoxic effects of the small dosages of cytotoxic drug (cisplatin or oxaliplatin) with the offered method cancer disease treatment is that the offered method cancer disease treatment leads to depression of cancer tumor metabolism due to suppression anabolic processes, i.e. suppression of mismatch repair (MMR) function via partial expression HSP90 function. However the peculiarity of cancer treatment mechanism confirms that the advantage of the combination very light cytotoxic effects of the small dosages of cytotoxic drug (cisplatin or oxaliplatin) with the offered method cancer disease treatment is similar 
to above described mechanism suppression depressed cancer metabolism with small dosage of cytotoxic drugs over suppression active cancer metabolism with great dosage cytotoxic drugs in up-to-date methods of cancer therapy.

Role of MMR (human MLH1(-) and MMR-deficient in cellular responses to 5-fluorouracil and 5-fluoro-2'-deoxyuridine (FdUrd)

Meyers M, et al. [36] investigated the role of MMR (human MLH1(-) and MMR-deficient of HCT116 colon cancer cells) in cellular responses to 5-fluorouracil and 5-fluoro-2'deoxyuridine (FdUrd). They have determined that HCT116 3-6 cells treated with a low dose of FdUrd have a 2-fold: greater G2 cell cycle arrested with MMR-deficient of HCT116 cells compared to enhanced G2 cell cycle in MMRproficient cells in response to cytotoxic agents.

a) Mechanisms of experiments outcomes: A low dose of FdUrd had a 2-fold: G2 cell cycle is arrested with MMRdeficient owing to distribution of cytotoxic effect with MMRdeficient of HCT116 cells among the all mismatch repair (MMR) proteins, unlike enhanced G2 cell cycle in MMRproficient cells in response to cytotoxic agents. However remote cellular reactions set cells to accept the substance of cytotoxic drug. Also remote cellular reactions cause attraction between cells and substance of cytotoxic drugs which can be depressed in MLH1-deficient tumor cells more than in MLH1-proficient tumor cells due to violation of link nucleus capacitors - mitochondria capacitors influencing on cellular capacitors [9, 10]. Therefore the remote cellular reaction between cells and cytotoxic drugs with MLH1-proficient tumor cells can be more absolute than with MLH1-deficient tumor cells. Hence the cytotoxic drugs in contact reaction with MLH1-proficient tumor cells can show greater cytotoxic effect than with MLH1-deficient tumor cells. Thus MLH1-deficient tumor cells can be less susceptible to cytotoxic drug and to apoptosis than MLH1proficient tumor cells, i.e. drug resistance occurs more in MLH1-deficient tumor cells.

b) Footnote: The advantage of the combination very light cytotoxic effects of a low dose of cytotoxic drug (FdUrd) with the offered method cancer disease treatment is that the offered method cancer disease treatment leads to depression of cancer tumor metabolism due to suppression anabolic processes, i.e. suppression of mismatch repair (MMR) function via partial expression catabolic function for cells survival. However it can affirm that the advantage of the combination very light cytotoxic effects of the small dosages of cytotoxic drug (FdUrd) with the offered method cancer disease treatment is similar to above described mechanism destruction depressed cancer metabolism with small dosage of cytotoxic drugs over suppression active cancer metabolism with great dosage cytotoxic drugs in up-to-date methods of cancer therapy.

Role bypass of DNA replication pathway in drug resistance mediated by loss of MMR

The interesting investigations were made by Moreland NJ, et al. [37]. They have used aphidicolin (Ap), an inhibitor of DNA polymerases, to study the role bypass of DNA replication pathway in drug resistance mediated by loss of MMR and received aphidicolin (Ap) sensitizing drugresistant cancer cells, that have lost MMR. Just their experiments showed bypass of DNA replication pathway in drug resistance in situation of loss of MMR, i.e. availability for drug resistance.

a) Mechanisms of experiments outcomes: The outcomes of these experiments confirm that the DNA reparative function by loss of MMR and received aphidicolin (Ap) (an inhibitor of DNA polymerases) occurs via bypass of DNA replication pathway and can be connected as with functional relationship between catabolic pathway and anabolic MLH1 proliferative functions, as well as with function of cellular capacitors $[9,10]$, i.e. drug resistance depending of cellular remote reactions, mediated by loss of MMR, which displays aphidicolin (Ap) drug-resistant mechanism by sensitizing cancer cells. These cellular remote reactions transit into contact biochemical reactions causing inhibition of DNA polymerases, i.e. the described mechanisms of bypass of DNA replication pathway in drug resistance in situation of loss of MMR.

b) Footnote: The advantage of the combination very light cytotoxic effects of the small dosage cytotoxic drug with the offered method cancer disease treatment is that the offered method cancer disease treatment leads to depression of cancer tumor metabolism due to suppression anabolic processes, i.e. suppression of mismatch repair (MMR) function and expression catabolic pathway. It was exhibited in Moreland NJ et al. experiments [37] with the mediated drug resistance by loss of MMR and received aphidicolin (Ap) sensitizing drug-resistant cancer cells via inhibition of DNA polymerases. However it can affirm that the advantage of the combination very light cytotoxic effects of the small dosages of cytotoxic drug with the offered method cancer disease treatment is similar to above described mechanism destruction depressed cancer metabolism with small dosage of cytotoxic drugs over suppression active cancer metabolism with great dosage cytotoxic drugs in up-to-date methods of cancer therapy.

Role of DNA mismatch repair (MMR) function and p53 function in drugs resistance

Lin $X$ and Howell SB [38] experiments exhibit that DNA mismatch repair (MMR) function and p53 function are major determinants of the rate of cisplatin resistance: Loss either MMR or p53 alone increased the rate of resistance to cisplatin. Lin X, et al. [39] and Yanamadala S, et al. [40] experiments exhibits that inhibition of DNA polymerase zeta by suppression of the expressed REV3 subunit, which eliminates the increased rate of cisplatin resistance, observed in the MMR-deficient cells.

Moreover, Yanamadala S, et al. [40] expressed opinion that MMR proteins can bind to certain DNA lesions and signal p53 and create apoptosis by an unknown mechanism, using both alkylating agents and $\mathrm{H} 2 \mathrm{O} 2$ which caused significant inhibition of mRNA synthesis in MLH1-expressing but not in MLH1-deficient cells. They suggest a novel mechanism of MLH1 in the induced p53 and apoptosis by inhibiting RNA polymerase II-dependent transcription and influencing on damaged DNA templates. Also Stubbert LJ, et al. [41] indicate that the transcription-coupled nucleotide excision 
repair (TC-NER) play a prominent role in determining the sensitivity of tumor cells to cisplatin even in the absence of p53 and DNA mismatch repair.

a) Mechanisms of experiments outcomes: DNA polymerase zeta takes part in somatic hypermutation of immunoglobulin genes, i.e. pathologic anabolic processes. Unlike DNA polymerase zeta, the mismatch repair (MMR) function and p53 prevent pathologic anabolic processes of excessive proliferative processes, exerting development of normal cellular cycle via maintenance balanced anabolic and catabolic processes in G2/M phases of cellular cycle. Thus expression DNA polymerase zeta and expression mismatch repair (MMR) function or p53 cause different chemical potentials $(u)$ which create the cellular capacitors with inverse cellular operations $[8,10]$. Therefore inhibition DNA polymerase zeta eliminates the increased rate of cisplatin resistance observed in the MMR-deficient cells. On the contrary, loss of either MMR or p53 alone increases the rate of resistance to cisplatin.

b) Footnote: The advantage of the combination very light cytotoxic effects of the small dosage cytotoxic drug with the offered method cancer disease treatment is that the offered method cancer disease treatment leads to depression of cancer tumor metabolism due to suppression anabolic processes, i.e. suppression either mismatch repair (MMR) function or p53 function of tumor suppressor. The suppression either mismatch repair (MMR) function or p53 function of tumor suppressor causes distribution of the drugs cytotoxic effects among the all mismatch repair (MMR) proteins. Thus it can affirm that the advantage of the combination very light cytotoxic effects of the small dosages of cytotoxic drug (cisplatin) with the offered method cancer disease treatment is similar to above described mechanism suppression depressed cancer metabolism with small dosage of cytotoxic drugs over suppression active cancer metabolism with great dosage cytotoxic drugs in up-to-date methods of cancer therapy.

\section{Investigating of anticancer agents minor groove binders (MGBs)}

Investigating the interesting class of anticancer agents Minor groove binders (MGBs), Fedier A, et al. [42] have chosen brostallicin which is a synthetic O \pm -bromoacrylic MGB. DNA minor groove binders (MGBs) are a class of anticancer agents highly effective in variety of human cancers. Just the outcomes of their researches reveal that brostallicin-induced cytotoxicity does not depend on functional DNA MMR. Besides, Fedier A, et al. [42] note that brostallicin does not alkylate DNA per se but operate through the interaction with GSH/GST system. Moreover all tumor cells are characterized with higher glutathione (GSH) and glutathione-S-transferase (GST) levels, according to data Geroni, et al. [43]. Also glutathione (GSH) is found in cellular wall, and DST enzyme catalyzes GSH peroxydase activity which lead to the detoxification of lipid and nucleic acid hydroperoxides, according to data Waxman DJ [44]. Also Waxman DJ [44] notes that DST enzyme exhibit a ligand binding function, which involves the non-covalent binding such substrate as heme, bilirubin, various steroids, and some lipophilic anticancer drugs. These investigations show that cytotoxic effects of Minor groove binders (MGBs) of brostallicin does not depends on DNA mismatch repair (MMR) mechanisms [42], and the cytotoxic effect MGB occurs through GSH/GST system of cellular wall and cytoplasm $[43,44]$.

a) Mechanisms of experiments outcomes: Causing cytotoxic effects by Minor groove binders (MGBs) of brostallicin, the changed GSH/GST systems of cellular wall and cytoplasm change cellular chemical potentials $(u)$ of cellular capacitors $[9,10]$, which don't depends on DNA mismatch repair (MMR) mechanisms. Thus the cytotoxic effects of Minor groove binders (MGBs) of brostallicin take part in cellular remote reaction through GSH/GST systems of cellular wall and cytoplasm, which involved also in neutralisation of ROS/ $\mathrm{H}_{2} \mathrm{O}_{2}$ / free radical in quiescent $\mathrm{G}_{0}$ and $\mathrm{G} 1 / \mathrm{S}$ phases cellular cycle, suppressing as processes DNA replication in G2/M phases cellular cycle as well as tumor excessive proliferative processes [10]. Then the cellular remote reaction transits into contact reaction [9, 10], as for recognizing substance of cytotoxic drug as well as for exerting subsequent processes of resistance to cytotoxic drug.

b) Footnote: The advantage of the combination very light cytotoxic effects of the small dosage cytotoxic drug (brostallicin) with the offered method cancer disease treatment is that the offered method cancer disease leads to depression of cancer tumor metabolism due to suppression anabolic processes. The remote cellular reactions between depressed cells of cancer tumor and cytotoxic effect of brostallicin, which are accomplished due to system of cellular capacitors operation fulfilling interplay between nucleus capacitors, mitochondria capacitors and other organelle capacitors connecting with cellular capacitors [10], precede contact reactions cells on cytotoxic drug brostallicin [9, 10]. Remote cellular reactions set cells to accept the substance of cytotoxic drug brostallicin and cause attraction between cells and substance of brostallicin [9]. However the depressed cancer cells in condition of prolonged medical starvation need for supplemental substances for their metabolism and maintenance stability basophilic chemical potential of their cytoplasm $(\mu)$. Therefore remote reaction of depressed cancer cells in condition of prolonged medical starvation of the offered method cancer disease treatment don't cause drugs resistance. Thus the cytotoxic effects of the small dosage brostallicin in contact reaction become stronger in condition of cancer cells depression over the suppressed anabolic processes of cancer cells by great dosage cytotoxic drug in the state of cells activity in modern methods cancer therapy, which show the similar cytotoxic effects as on hormonal cells and immune cells of an organism as well as on tumor cells. Damage hormonal and immune defensive reactions of an organism can lead as to recurrence cancer disease after some medical remissions, i.e. palindromia of cancer development, and as well as to resistance to anticancer drugs as the result of intensive anticancer chemotherapy with great dosages of cytotoxic drugs in cancer therapy. Just the mechanisms of offered method cancer treatment don't damage immune and 
hormonal systems and don't cause drug resistance and recurrence cancer disease after some medical remissions.

Critical reviews of the mechanisms decrease of medical drugs efficiency in palindromia phase, i.e. recurrence disease after cancer disease remission, due to treatment with great dosage of cytotoxic drugs

General mechanism of decrease of medical drugs efficiency in palindromia: Suppression medical drugs activity occurs after multiple remissions of cancer disease, but suppression medical drugs activity does not occur in the beginning of the treatment by cytotoxic drugs. Thus mechanism of immune responses is appeared after remote reactions across distance via producing immune rearrangement in the immune cells due to multiple use anticancer drugs leading to cancer disease remission. Then remote reactions transit into contact phase of immune reactions which destroy these anticancer drugs causing decrease of medical drugs efficiency [9].

Role RAS/RAF/ERK pathway in drugs resistance: Piscazzi A, et al. [45] found that the essential activation of the RAS/RAF/ ERK pathway may lead to resistance to sunitinib in thyroid carcinoma cells, although sunitinib inhibits selectively cell proliferation inducing cell cumulation in the $\mathrm{G}_{0}-\mathrm{G}_{1}$ phase and inhibits the phosphorylation of ERK1/2 in both KRAS/ BRAF wild-type thyroid cancer cells and in tumor cells harboring the RET/PTC rearrangement. Furthermore they described that the activation of RAS/RAF/ERK signaling in KRAS/BRAF wild-type cells by transfection of the R12 HRAS or V600E BRAF mutants or stimulation with epithelial growth factor resulted in the loss of responsiveness to sunitinib, whereas pharmacological inhibition of MAPK kinase activity resulted in the reset sensitivity of KRAS- or BRAF-mutated cells to the multikinase inhibitor.

Also Tzu-Hurng Cheng, et al. [46] researches exhibit that RAS/RAF/ERK kinase pathway regulates extracellular signal due to mediated of cyclic strain by ROS. Extracellular signals induce endothelin-1 gene.

a) Footnote: Just ROS promote processes replication in G2 phase cellular cycle exerting process proliferation which is induced by endothelin-1 gene [10]. These excessive processes of cancer metabolism lead to misbalance between extracellular chemical potential ( $\left.\mu_{\text {extracell }}\right)$ and intracellular chemical potential $\left(\mu_{\text {intracell }}\right)$, due to excessive expression RAS/RAF extracellular proteins. This misbalance causes inflow substances from extracellular medium into intracellular medium /according to Theorell formula exerting G1/M phases cellular cycle for excessive cancer proliferative processes [3] (Figure 1). The influences of immune cells via their cellular capacitors operations on sunitinib molecule promote suppression of sunitinib activity and cause resistance to sunitinib via immune remote and contact reactions $[8,9]$. The Ras-Raf-MEK-ERK pathway or MARK/ERK pathway is formed owing to suppression cytotoxic effect of sunitinib $[8,9]$. Thus these investigations confirm the mechanism of excessive expression RAS/RAF/ ERK owing to immune cells reaction on cytotoxic drug substances after some remissions of cancer disease.
Interactions between gene amplification in cancer cells and immune response on cytotoxic drug in mechanisms of decreased drug efficiency

Oliveras-Ferraros C, et al. [47] evaluated the connection of HER2 gene amplification with resistance to the EGFR (HER2)-targeted antibody cetuximab and of response to combination therapies against EGFR and HER2 in wildtype KRAS tumor by patients with colorectal cancer. Then they concluded that cetuximab activity against cetuximabrefractory wild-type KRAS squamous cell carcinoma (SCC) cells can be fully restored in the presence of the anti-HER2 monoclonal antibody trastuzumab.

a) Footnote: Thus this investigation has determined the connected resisting mechanisms amplification of HER2 gene and immune response of the EGFR (HER2)-targeted antibody cetuximab in wild-type KRAS tumor of patients with colorectal cancer. It is quite true these interactions between gene amplification in cancer cells and immune response on cytotoxic drug in mechanisms of decreased drug efficiency. Really, cetuximab suppression due to immune cells reaction exerts gene amplification due to influence suppressed cytotoxic drug on DNA gene.

Clinical investigation of interactions between SKP2 gene amplification and SKP2 immune-expression

$\mathrm{Li}$ CF, et al. [48] studied clinical aggressiveness of myxofibrosarcoma and evaluated interactions between SKP2 gene amplification and SKP2 immune-expression for prognosis and independently predictive of this disease.

Footnote: Indeed the study of influences of immuneexpression on gene amplification is most important for clinical purposes regarding prognosis of cancer disease.

\section{Studies of epigenetic changes in cancer tumor}

Describing epigenetic changes in cancer tumors [49] expressed doubts: "Aberrant DNA methylation at CpG islands and associated epigenetic silencing are observed during the acquisition of drug resistance. However, it remains unclear whether all of the observed changes are drivers of drug resistance, causally associated with response of tumours to chemotherapy, or are passenger events representing chance DNA methylation changes".

Footnote: The changes in cancer cells DNA including epigenetic changes could appear at current cellular cycle phases of cancer development due to oncogene influences, which are subjected to immune responses due to cellular capacitors of immune cells operations via remote cellular reactions, owing to resonance waves, transiting into contact cellular reactions and suppressing cytotoxic drug (as strange object), that results in epigenetic changes in DNA of cancer cells owing to influence of suppressed cytotoxic drugs. Thus it occurs resistance to cytotoxic drugs after a phase of cancer disease remission and lead to recurrence cancer disease [8-10].

Role of extracellular superoxide dismutase (ECSOD) in cancer metabolism

Studying the role of extracellular superoxide dismutase 
(EcSOD) in lung cancer, Teoh-Fitzgerald ML, et al., [50] found that air respiration pathway in normal epithelial cells expressed abundant EcSOD and have an unmethylated promoter, whereas lung cancer cells displayed aberrant promoter hypermethylation and decreased chromatin accessibility. EcSOD is considerably lower in lung tumors than in normal tissue, and EcSOD promoter is hypermethylated in adenocarcinomas as compared with normal lungs. Furthermore re-expression of EcSOD attenuates malignant phenotype of lung carcinoma cells, decreasing invasion and survival considerably. However loss of EcSOD expression in lung cancer is the result of ECSOD promoter methylation and loss of heterozygosity (LOH) of EcSOD. It was suggested that its early loss may contribute to extracellular matrix (ECM) remodeling and malignant progression.

Footnote: It is known that ROS/ $\mathrm{H}_{2} \mathrm{O}_{2}$ / free radicals create cellular replication in $\mathrm{G} 2$ phase cellular cycle causing proliferative processes [10, 12]. However extracellular superoxide dismutase (EcSOD) does not suppress capability of intracellular superoxide dismutase causing dismutation of intracellular superoxide anions. Just prolonged treatment with cytotoxic drugs leads to the epigenetic changes of EcSOD hypermethylation causing by hypermethylated EcSOD promoter. The epigenetic inactivation of extracellular superoxide dismutase (EcSOD), due to aberrant promoter hypermethylation operation, should activate intracellular superoxide dismutase maintaining difference chemical potentials and charges on inner and outer cellular membranes for cellular capacitors operation [9]. Intracellular superoxide dismutase exerts processes DNA replication due to promoting intracellular dismutation of superoxide $\left[\mathrm{O}_{2}{ }^{*}-\right]$ converting superoxide into hydrogen peroxide $\left[\mathrm{H}_{2} \mathrm{O}_{2}\right]$ and following into free radicals, which advance $\mathrm{G} 2$ phase cellular cycles of cancer cells $[9,10,12]$. The epigenetic changes can appear as the result of immune responses on cytotoxic drug which lead to violation of cytotoxic drugs. Then such cytotoxic drugs cause loss of EcSOD due to EcSOD promoter methylation and loss of heterozygosity ( $\mathrm{LOH}$ ) of EcSOD which lead to malignant progression and drugs resistance after a phase of cancer disease remission, causing recurrence cancer disease.

The investigations of role epigenetic changes in decrease cancer efficiency

Investigating operations of several cytotoxic drugs as azacitidine (Vidaza), decitabine (Dacogen), vorinostat (Zolinza), and romidepsin (Istodax), Boumber $Y$ and Issa JP [51] inquired: "Epigenetics in cancer: what's the futurea". Describing expected results of these drugs combination in clinical trials with DNA methylation inhibitors and histone deacetylase inhibitors, the authors expressed doubt: "unclear mechanisms of response and resistance, and rare responses in solid tumors".

a) Footnote: Just the mechanism decrease drugs efficiency after intensive chemotherapy with great dosage cytotoxic drugs and multiple remissions, causing epigenetic changes, depends on immune cells remote reactions on these drugs and epigenetic changes due to cellular remote reactions of immune cells on intensive cytotoxic chemotherapy. Indeed cellular capacitors of immune cells react on cytotoxic drugs and cause suppression them. The suppressed cytotoxic drugs cause epigenetic changes in cancer nuclei, creating rearrangement nucleus DNA chemical structure of cancer cells. On the other hand, immune cells become sensitive to cytotoxic drugs causing suppression of cytotoxic drugs and drugs resistance after cancer disease remission.

\section{Investigations of targeting cancer tumor by tumor suppressor's genes}

Gramling S, et al. [52] noted that pharmacologically targeting cancer tumor by tumor suppressor genes has not been fruitful, because many tumor suppressors genes and oncogenes are irreversibly both commonly altered or entirely deleted during carcinogenesis, thereby making it difficult to restore gene function.

Footnote: Indeed many tumor suppressor's genes and oncogenes operate as primers of oncogenesis. Then the oncogenesis advances according to Glansdorff and Prigogine theory resulting in non-linear pathologic development thermodynamic system of an organism [8]. They can cause small counteractions of anticancer drugs efficiency. However these counteractions are not mechanisms of cancer drug resistance after multiple remissions of cancer disease, but such suppression medical drug can occur only in the beginning of the chemotherapy treatment. Furthermore the other such samples as putative tumor suppressor gene, anticancer protein BRM, a key SWI/SNF chromatic remodeling complex subunit, which direct and limit the execution of specific cellular programs such as differentiation and growth control, are inactivated in many tumor types according to Gramling $S$, et al. data [52]. Hence Gramling S, et al. reply that targeting tumor suppressor genes is not fruitful [52]. Thus the mechanisms of tumor suppressor's miRNAs, as well as the other tumor suppressors, cannot be the main cause decreasing cancer drugs efficiency after multiple remissions of cancer disease. Only suppressed cancer development die to use of offered "Polonged medical starvation", which is also treated by deceased dosage cytotoxic drugs, prevents as resistance to cytotoxoc drugs as well as recurrence cancer disease after some disease remissions.

\section{Investigations of participation immune mechanisms in decrease cancer efficiency}

Studying antitumor efficiency of cisplatin LiX, et al. [53] have noted:"despite of extraordinaryactivities of cisplatin against a variety of solid tumors, cis-Dichlorodiamminoplatinum (II) (cisplatin)has demonstrated capability to reduce the toxicity and enhance the circulation time of cisplatin in the clinical efficacy. It was defined that cisplatin was incorporated into the nanoparticles with high encapsulation efficiency more than $75 \%$ and core-shell structure nanoparticles were prepared from block copolymer of methoxy poly(ethylene glycol)-polycaprolactone (mPEG-PCL). Then Li X, et al. [53] concluded that cisplatin-loaded nanoparticles exhibited superior antitumor effect by delaying tumor growth. When this drug was delivered intratumorally, significant 
improvement was not observed. Therefore cisplatin was administrated intraperitoneally as opposed to action of free cisplatin.

Footnote: Thus these investigations prove participation peritoneal immune mechanism of reticuloendothelial system in action of cisplain decreased for anticancer efficiency.

\section{Acknowledgments}

This article is dedicated to the memory of my daughter T.M. Ponisovska.

\section{Conflict of interest}

The author declares no conflict of interest.

\section{References}

[1] Breuss R. The cancer, leucomia and the other diseases", edit. Logos, 1992.

[2] Breuss R. The Breuss Cancer Cure, Alive books Canada, 1995.

[3] Ponisovskiy MR. Cancer metabolism and the Warburg effect as anabolic process outcomes of oncogene operation. Crit Rev Eukaryot Gene Expr. 2010; 20(4):325-339.

[4] Ponisovskiy MR. Warburg effect mechanism as the target for theoretical substantiation of a new potential cancer treatment. Crit Rev Eukaryot Gene Expr. 2011; 21(1):13-28.

[5] Ponizovskiy MR. The detailed description mechanisms of the herbs extracts operations in the new method cancer disease treatment via rearrangement of metabolism from pathologic development into normal development. J Clin Trials. 2012; 2:124.

[6] Ponizovskiy MR. The Central Regulation of all Biophysical and Biochemical Processes as the Mechanism of Maintenance Stability of Internal Energy and Internal Medium both in a Human Organism and in cells of an Organism. Mod Chem appl. 2013; 1:e101.

[7] Ponizovskiy MR. The mechanisms maintenance stability Internal Energy and Internal Medium an organism in norm and in quasistationary pathologic states. Biochem Physiol. 2013; 2:115.

[8] Ponizovskiy MR. Biophysical and biochemical models of mechanisms of cellular development via the cellular cycle in normal tissue, cancerous tissue, and inflammatory processes. Crit Rev Eukaryot Gene Expr. 2013; 23(2):171-193.

[9] Ponisovskiy MR. Driving mechanisms of passive and active transport across cellular membranes as the mechanisms of cell metabolism and development as well as the mechanisms of cellular distance reactions on hormonal expression and the immune response. Crit Rev Eukaryot Gene Expr. 2011; 21(3):267-290.

[10] Ponizovskiy MR. Biophysical and biochemical transmutation of mitochondrial function in cancer genesis. Biochem Anal Biochem. 2013; 2(3).

[11] Tan AS, Baty JW, Dong LF, Bezawork-Geleta A, Endaya B, et al. Mitochondrial genome acquisition restores respiratory function and tumorigenic potential of cancer cells without mitochondrial DNA. Cell Metab. 2015; 21(1):81-94.

[12] Furda Amy Marie. The role of mtDNA damage in mitochondrial dysfunction. University of Pittsburg. 2011; pp.145.

[13] Gibellini L, Pinti M, Nasi M, De Biasi S, Roat E, et al. Interfering with ROS Metabolism in Cancer Cells: The Potential Role of Quercetin. Cancers (Basel). 2010; 2(2):1288-1311.

[14] Szatrowski TP, Nathan CF. Production of large amounts of hydrogen peroxide by human tumor cells. Cancer Res. 1991; 51(3):794-798.

[15] Westermann B. Mitochondrial fusion and fission in cell life and death. Nat Rev Mol Cell Biol. 2010; 872-884.

[16] lyer RR, Pluciennik A, Burdett V, Modrich PL. DNA mismatch repair: functions and mechanisms. Chem Rev. 2006; 106(2):302-323.

[17] Larrea AA, Lujan SA, Kunkel TA. DNA mismatch repair. Cell. 2010; 141(4):730.
[18] Li GM. Mechanisms and functions of DNA mismatch repair. Cell Res. 2008; 18(1):85-98.

[19] Palacios-Callender M, Hollis V, Mitchison M, Frakich N, Unitt D, et al. Cytochrome $c$ oxidase regulates endogenous nitric oxide availability in respiring cells: a possible explanation for hypoxic vasodilation. Proc Natl Acad Sci U S A. 2007; 104(47):18508-18513.

[20] Radi R. Nitric oxide, oxidants, and protein tyrosine nitration. Proc Natl Acad Sci U S A. 2004; 101(12):4003-4008.

[21] Brookes PS, Levonen AL, Shiva S, Sarti P, Darley-Usmar VM. Mitochondria: regulators of signal transduction by reactive oxygen and nitrogen species. Free Radic Biol Med. 2002; 33(6):755-764.

[22] Krock BL, Skuli N, Simon MC. Hypoxia-induced angiogenesis: good and evil. Genes Cancer. 2011; 2(12):1117-1133.

[23] Shipard Isabell. How can I use Herbs in my daily life $\mathrm{G}$ Grassroot. 2013.

[24] Helfer M, Koppensteiner H, Schneider M, Rebensburg S, Forcisi S, et al. The root extract of the medicinal plant Pelargonium sidoides is a potent HIV-1 attachment inhibitor. PLoS One. 2014; 9(1):e87487.

[25] Imesch P, Goerens A, Fink D, Fedier A. MLH1-deficient HCT116 colon tumor cells exhibit resistance to the cytostatic and cytotoxic effect of the poly(A) polymerase inhibitor cordycepin (3'-deoxyadenosine) in vitro. Oncol Lett. 2012; 3(2):441-444.

[26] Fedier A, Poyet C, Perucchini D, Boulikas T, Fink D. MLH1-deficient tumor cells are resistant to lipoplatin, but retain sensitivity to lipoxal. Anticancer Drugs. 2006; 17(3):315-323.

[27] Sergent C, Franco N, Chapusot C, Lizard-Nacol S, Isambert N, et al. Human colon cancer cells surviving high doses of cisplatin or oxaliplatin in vitro are not defective in DNA mismatch repair proteins. Cancer Chemother Pharmacol. 2002; 49(6):445-452.

[28] Ohta T, Ohmichi M, Hayasaka T, Mabuchi S, Saitoh M, et al. Inhibition of phosphatidylinositol 3-kinase increases efficacy of cisplatin in in vivo ovarian cancer models. Endocrinology. 2006; 147(4):1761-1769.

[29] Stathopoulos GP, Boulikas T. Lipoplatin formulation review article. J Drug Deliv. 2012; 2012:581363.

[30] Fedier A, Erdmann R, Boulikas T, Fink D. Potential of the Akt inhibitor LY294005 to antagonize the efficacy of Cisplatin against HCT116 tumor cells in a DNA mismatch repair-dependent manner. Int J Oncol. 2006; 29(5):1303-1310.

[31] Elstrom RL, Bauer DE, Buzzai $M$, Karnauskas $R$, Harris $M H$, et al. Akt stimulates aerobic glycolysis in cancer cells. Cancer Res. 2004; 64(11):3892-3899.

[32] Gottlob K, Majewski N, Kennedy S, Kandel E, Robey RB, et al. Inhibition of early apoptotic events by Akt/PKB is dependent on the first committed step of glycolysis and mitochondrial hexokinase. Genes Dev. 2001; 15(11):1406-1418.

[33] Plas DR, Talapatra S, Edinger AL, Rathmell JC, Thompson CB. Akt and $\mathrm{BCl}-\mathrm{xL}$ promote growth factor-independent survival through distinct effects on mitochondrial physiology. J Biol Chem. 2001; 276(15):1204112048.

[34] Plas DR, Thompson CB. Akt-dependent transformation: there is more to growth than just surviving. Oncogene. 2005; 24(50):7435-7442.

[35] Fedier A, Stuedli A, Fink D. Presence of MLH1 protein aggravates the potential of the HSP90 inhibitor radicicol to sensitize tumor cells to cisplatin. Int J Oncol. 2005; 27(6):1697-1705.

[36] Meyers M, Wagner MW, Hwang HS, Kinsella TJ, Boothman DA. Role of the hMLH1 DNA mismatch repair protein in fluoropyrimidine-mediated cell death and cell cycle responses. Cancer Res. 2001; 61(13):51936201.

[37] Moreland NJ, Illand M, Kim YT, Paul J, Brown R. Modulation of drug resistance mediated by loss of mismatch repair by the DNA polymerase inhibitor aphidicolin. Cancer Res. 1999; 59(9):2102-2106.

[38] Lin X, Howell SB. DNA mismatch repair and p53 function are major determinants of the rate of development of cisplatin resistance. Mol Cancer Ther. 2006; 5(5):1239-1247.

[39] Lin X, Ramamurthi K, Mishima M, Kondo A, Christen RD, et al. P53 modulates the effect of loss of DNA mismatch repair on the sensitivity of human colon cancer cells to the cytotoxic and mutagenic effects of cisplatin. Cancer Res. 2001; 61(4):1508-1516.

[40] Yanamadala S, Ljungman M. Potential role of MLH1 in the induction of p53 and apoptosis by blocking transcription on damaged DNA templates. Mol Cancer Res. 2003; 1(10):747-754.

[41] Stubbert LJ, Smith JM, McKay BC. Decreased transcription-coupled nucleotide excision repair capacity is associated with increased p53and MLH1-independent apoptosis in response to cisplatin. BMC Cancer. 2010; 10:207. 
[42] Fedier A, Fowst C, Tursi J, Geroni C, Haller U, et al. Brostallicin (PNU166196)--a new DNA minor groove binder that retains sensitivity in DNA mismatch repair-deficient tumour cells. Br J Cancer. 2003; 89(8):1559-1565.

[43] Geroni C, Marchini S, Cozzi P, Galliera E, Ragg E, et al. Brostallicin, a novel anticancer agent whose activity is enhanced upon binding to gluthathione. Cancer Res. 2002; 62(8):2332-2336.

[44] Waxman DJ. Glutathione S-transferases: role in alkylating agent resistance and possible target for modulation chemotherapy--a review. Cancer Res. 1990; 50(20):6449-6454.

[45] Piscazzi A, Costantino E, Maddalena F, Natalicchio MI, Gerardi AM, et al. Activation of the RAS/RAF/ERK signaling pathway contributes to resistance to sunitinib in thyroid carcinoma cell lines. J Clin Endocrinol Metab. 2012; 97(6):E898-906.

[46] Cheng TH, Shih NL, Chen SY, Loh SH, Cheng PY, et al. Reactive oxygen species mediate cyclic strain-induced endothelin-1 gene expression via RAS/RAF extracellular signal-regulated kinase pathway in endothelian cells. J Mol Cell Cardiol. 2001; 33(10):1805-1814.

[47] Oliveras-Ferraros C, Massaguer Vall-Llovera A, Vazquez-Martin A, Salip DC, Queralt B, et al. Transcriptional upregulation of HER2 expression in the absence of HER2 gene amplification results in cetuximab resistance that is reversed by trastuzumab treatment. Oncol Rep. 2012; 27(6):1887-1892.

[48] Li CF, Wang JM, Kang HY, Huang CK, Wang JW, et al. Characterization of gene amplification-driven SKP2 overexpression in myxofibrosarcoma: potential implications in tumor progression and therapeutics. Clin Cancer Res. 2012; 18(6):1598-1610.

[49] Zeller C, Brown R. Therapeutic modulation of epigenetic drivers of drug resistance in ovarian cancer. Ther Adv Med Oncol. 2010; 2(5):319329.

[50] Teoh-Fitzgerald ML, Fitzgerald MP, Jensen TJ, Futscher BW, Domann FE. Genetic and epigenetic inactivation of extracellular superoxide dismutase promotes an invasive phenotype in human lung cancer by disrupting ECM homeostasis. Mol Cancer Res. 2012; 10(1):40-51.

[51] Boumber Y, Issa JP. Epigenetics in cancer: what's the future 0 Oncology (Williston Park). 2011; 25(3):220-226.

[52] Gramling S, Rogers C, Liu G, Reisman D. Pharmacologic reversal of epigenetic silencing of the anticancer protein BRM: a novel targeted treatment strategy. Oncogene. 2011; 30(29):3289-3294.

[53] Li X, Li R, Qian X, Ding Y, Tu Y, et al. Superior antitumor efficiency of cisplatin-loaded nanoparticles by intratumoral delivery with decreased tumor metabolism rate. Eur J Pharm Biopharm. 2008; 70(3):726-734. 